\title{
Efforts to overcome hypoxia condition in Balb/c mouse macrophages after intraperitoneal SRBC immunization
}

\author{
Pungguri Ayu Nega Sarsanti, ${ }^{1}$ Mohamad Sadikin, ${ }^{2,3}$ Sri Widia Azraki Jusman ${ }^{2,3}$
}

pISSN: 0853-1773 • elSSN: 2252-8083 https://doi.org/10.13181/mji.v28i1.1961 Med J Indones. 2019;28:14-20

Received: April 24, 2017

Accepted: January 17, 2019

Author's affiliations:

${ }^{1}$ Biomedical Sciences Graduate Program, Faculty of Medicine, Universitas Indonesia, Jakarta, Indonesia, ${ }^{2}$ Center of Hypoxia and Oxidative Stress Study, Faculty of Medicine, Universitas Indonesia, Jakarta, Indonesia, ${ }^{3}$ Department of Biochemistry and Molecular Biology, Faculty of Medicine, Universitas Indonesia, Jakarta, Indonesia

\section{Corresponding author:}

Mohamad Sadikin

Department of Biochemistry and Molecular Biology, Faculty of Medicine, Universitas Indonesia, Jalan Salemba 6, Senen, Central Jakarta 10320, DKI Jakarta, Indonesia

Tel/Fax: +62-21-3910734/

+62-21-3910190

E-mail: sadikinmohamad@gmail.com

\begin{abstract}
BACKGROUND Activated macrophages require increased oxygen to destroy foreign bodies, leading to an increase in the levels of reactive oxygen species (ROS). Therefore, macrophages would experience hypoxic and oxidative stress conditions at the same time. Thus, this study was aimed to evaluate the mechanism of the activated macrophages to overcoming this dual condition.
\end{abstract}

METHODS The activated macrophages were harvested from the intraperitoneal cavities of $18 \mathrm{BALB} / \mathrm{C}$ mice immunized with $2 \%$ sheep red blood cells (SRBCs). The macrophage suspension was divided into four groups: control, 24, 48, and 72 hours after-immunization groups. The expressions of hypoxia-inducible factor (HIF)-1a, HIF-2a, and cytoglobin (Cygb), as markers for hypoxic condition, were measured by quantitative reverse transcription polymerase chain reaction (qRT-PCR) and enzymelinked immunosorbent assay (ELISA), whereas peroxisome proliferator-activated receptor gamma coactivator (PGC-1a) protein as a marker for mitochondrial biogenesis and aerobic metabolism was measured with ELISA. The analysis of oxidative stress was conducted with the water-soluble tetrazolium salt test.

RESULTS The HIF-1a mRNA expression was the highest at 24 hours, whereas the HIF2a mRNA showed no increased expression during the observation. The Cygb mRNA decreased after 24 hours. The highest expressions of HIF-1a and HIF-2a proteins were detected at 72 hours, whereas the Cygb protein expression increased since 24 hours. The PGC-1a protein expression increased at 72 hours. The WST test showed the highest ROS level at 24 hours.

CONCLUSIONS The macrophages were activated by SRBCs underwent dual hypoxia and oxidative stress condition simultaneously to overcome the foreign bodies. The macrophages overcame these stress conditions by increasing their aerobic metabolism.

KEYWORDS cytoglobin, HIF-1a, HIF-2a, hypoxia, macrophage, PGC-1a
Macrophages are the cells involved in the immune response and are widespread in various tissues, including the peritoneum.' Macrophages play an important role in the antigen destruction through oxygen burst and phagocytosis. Macrophages are important in the antigen-presenting process to lymphocytes. ${ }^{2}$ Therefore, macrophages are essential to connecting the innate and adaptive immunities. Macrophages engulf and destroy foreign bodies, such as bacteria, into phagosomes. ${ }^{3}$
The foreign bodies will be destroyed by oxidation and hydrolysis. The oxidation of foreign bodies requires reactive oxygen species (ROS) in the form of anion superoxide, which will be converted by hydrogen peroxide. The peroxide will be transformed into hypochlorous acid ( $\mathrm{HOCl})$, which acts directly on foreign macromolecules. ${ }^{4}$ For this objective, macrophages need large quantities of oxygen. The phagosomes will fuse with the lysosomes, which contain hydrolase enzymes 
that can destroy the foreign body macromolecules. Lysosomes contain a variety of enzymes, such as esterases, glucuronidases, glycosidases, cathepsins, ribonucleases, and deoxyribonucleases. ${ }^{5}$

On the other hand, the macrophages will synthesize and secrete various types of protein that act as cytokines. All these processes require a large amount of oxygen, which cannot be provided through the existing general mechanism of cells. This condition causes the cells to undergo relative hypoxia. ${ }^{6}$ To overcome this condition, the cells are equipped with transcription factors, i.e., hypoxia-inducible factors (HIFs). HIFs exist in three forms: HIF-1, HIF-2, and HIF-3.7 Each factor consists of two subunits: $\alpha$ and $\beta$. The $a$ subunit is specific for a type of HIF, whereas the $\beta$ subunit is the same for all HIF types. HIF-1 and HIF-2 are widely studied, whereas the role of HIF-3 remains unclear.

In cells, the entering oxygen should be distributed properly, especially to the organelles, i.e., mitochondria, which consume this molecule in large quantities. Oxygen can only enter the cells in the form of a complex with another molecule. This form is realized by a special protein, the hemoglobin $(\mathrm{Hb})$, which is found in erythrocytes. Since long ago, an extra erythrocyte type of Hb, i.e., myoglobin, was found in all type of striated muscles. Early in this century, the second type of extracellular $\mathrm{Hb}$, neuroglobin (Ngb), was detected in the brain. After several years, the third type of extra erythrocyte $\mathrm{Hb}$, cytoglobin (Cygb), was discovered. This protein is found in practically all cells other than the striated muscles and the nervous system. ${ }^{8}$ Supposedly, Cygb captures the $\mathrm{O}_{2}$ released by erythrocyte $\mathrm{Hb}$ and transfers the molecule to the mitochondria. Several studies suggested that HIF-1 can upregulate the expression of the mRNA and protein of Cygb. ${ }^{9}$

Activated macrophages require increased oxygen levels to destroy foreign bodies, leading to an increase in ROS. ${ }^{10}$ Therefore, the macrophages would experience hypoxic and oxidative stress conditions at the same time. To date, limited evidence proves this dual condition. Thus, this study was aimed to evaluate the mechanism of activated macrophages in overcoming the dual hypoxic and oxidative stress condition.

\section{METHODS}

\section{Animals}

The $B A L B / C$ mice were obtained from the Primate Study Center, IPB, Bogor and acclimated in the laboratory conditions for 2 weeks and fed with standard diet ad libitum. A total of 24 male mice were divided randomly and equally into four groups. The first was the control, non-immunized group. The second, third, and fourth groups were immunized with sheep red blood cells (SRBCs), and their peritoneal macrophages were harvested 24,48 , and 72 hours after immunization, respectively. All the protocols in this study were reviewed and agreed by the Research Ethical Committee of Faculty of Medicine, Universitas Indonesia number 292/UN2.F1/ETIK/2016.

\section{Immunization method}

The SRBCs were used as antigens and obtained from the Department of Microbiology FMUI. The SRBCs were collected from sheep by jugular vein function. The SRBCs were placed in acid citrate dextrose. The blood collected was centrifuged at 1,000 $\mathrm{g}$ for $10 \mathrm{~min}$. The buffy coat containing leukocytes was removed, and the remaining SRBCs were washed thrice extensively with $0.9 \% \mathrm{NaCl}$. The $2 \% \mathrm{SRBC}$ suspension was prepared in normal saline, and $0.2 \mathrm{ml}$ of this suspension was injected intraperitoneally to each mouse, except the control group.

\section{Macrophage separation}

To collect the intraperitoneal macrophages, each mouse was injected intraperitoneally with $5 \mathrm{ml}$ cold phosphate-buffered saline (PBS). This operation was conducted after euthanasia of the corresponding mice by cervical dislocation. After injection, the abdominal wall was massaged gently, and the peritoneal cells were collected by aspiration of the cold PBS within the peritoneal cavity with Pasteur pipettes. The collected cells were centrifuged at $176 \mathrm{~g}$ (1,500 rpm). The supernatant was discarded, and the precipitated cells were resuspended in $1 \mathrm{ml}$ PBS. The number of cells in the suspension was counted with the improved Neubauer chamber. The macrophages were separated from the other cells by the adherence method..$^{11,12}$

\section{Phagocytosis experiment}

A total of 200 macrophages in Roswell Park Memorial Institute medium were plated on cover slips placed within $5 \mathrm{~cm}$ diameter of the Petri dishes, followed by the addition of $3 \mu$ lipopolysaccharides (LPS). After $30 \mathrm{~min}$ of incubation, the SRBCs were added in proportion of 10 cells for each macrophage. All the mixtures in the Petri dishes were incubated in a 
moisture chamber at $37^{\circ} \mathrm{C}$ for $30 \mathrm{~min}$. After this period, the cover slips were removed from the Petri dish and dried in air, followed by methanol fixation. The cover slips were stained with $3 \%$ Giemsa staining solution for $15 \mathrm{~min}$ and washed by distilled water. The cover slips were mounted on the object glass and examined under the microscope with 400 magnifications.

\section{Water-soluble tetrazolium salt (WST) assay}

The WST assay was used to calculate the concentration of superoxide anion. The macrophage monolayer in the microtube was overlaid with $1 \mathrm{ml}$ Hank's solution, followed by the addition of $2 \mu \mathrm{l}$ WST. This step was followed by an incubation period of $4 \mathrm{~h}$ at room temperature. Then, the supernatant was removed and used to determine the WST reduction by reading the optical density (OD) with a spectrophotometer at $450 \mathrm{~nm}$ against distilled water as the blank. A higher OD, as obtained in the absorbance reading, was associated with higher amounts of WST reduction and thus of superoxide anions generated by the macrophages.

\section{Reverse transcription polymerase chain reaction (RT- PCR)}

The HIF-1a, HIF-2a, and Cygb mRNA expressions were measured using RT-PCR method. The reagents needed included the primer, RNA, KAPA SYBR Fast, and nuclease-free water. The principle of RT-PCR is converting mRNA into CDNA and then replicating the CDNA via PCR cycles that have been set. Table 1 presents the primer sequences used in this study. $\beta$-actin, HIF-1a, and HIF-2 $\alpha$ primers were designed using NCBI.

The mRNA was isolated from the macrophages after 2 cycles of freezing and thawing in $0.5 \mathrm{ml}$ microtube, followed by the addition of $0.8 \mathrm{ml}$ TriPure Isolation Reagent (Roche). Then, the RNA was isolated according to the procedure described in the manual included in the kit. The relative expressions of mRNAs were realized using real time RT-PCR (EcoSains). Each reaction mixture contained $5 \mu \mathrm{l}$ KAPA SYBR Fast master mix, $0.2 \mu \mathrm{l}$ KAPA-RT mix, $0.2 \mu \mathrm{l}$ forward primer, $0.2 \mu \mathrm{l}$ reverse primer, RNA template (equivalent to 100 ng RNA), and nuclease-free water, achieving a total volume of $10 \mu$ l.

The $P C R$ reaction was conducted using KAPA SYBR Fast One-Step qRT-PCR kit. According to the procedure, CDNA was synthesized at $42^{\circ} \mathrm{C}$ for $5 \mathrm{~min}$ and KAPA RT mix inactivated at $95^{\circ} \mathrm{C}$ for $5 \mathrm{~min}$. The PCR cycles and detection ( 45 cycles) lasted for $10 \mathrm{~s}$ at $95^{\circ} \mathrm{C}$,
Table 1. Primer sequences used for PCR

\begin{tabular}{ll}
\hline Gene & \multicolumn{1}{c}{ Primer } \\
\hline -actin & F: 5'-CTA AGG CCA ACC GTG AAA AG-3' \\
& R: 5'-ACC AGA GGC ATA CAG GGA CA-3' \\
HIF-1 $\alpha$ & F: 5'-GCA CTA GAC AAA GTT CAC CTG AGA-3' \\
& R: 5'-CGC TAT CCA CAT CAA AGC AA-3' \\
HIF-2 $\alpha$ & F: 5'-CTC CAG GAG CTC AAA AGG TG-3' \\
& R: 5'-CAG GTA AGG CTC GAA CGA TG-3' \\
Cygb $^{13}$ & F: 5'-CGC AGC CTA CAA GGA AGT G-3' \\
& R: 5'-CCT GAA GAG GGC AGA GTG G-3' \\
\hline
\end{tabular}

$\mathrm{HIF}=$ hypoxia-inducible factor; $\mathrm{Cygb}=$ cytoglobin

$40 \mathrm{~s}$ at $52-54^{\circ} \mathrm{C}$, and $30 \mathrm{~s}$ at $72^{\circ} \mathrm{C}$. Melting curve analysis was conducted for $15 \mathrm{~S}$ at $95^{\circ} \mathrm{C}, 15 \mathrm{~S}$ at $55^{\circ} \mathrm{C}$, and $15 \mathrm{~S}$ at $55^{\circ} \mathrm{C}$. Then, the samples were incubated for $2 \mathrm{~min}$ at $50^{\circ} \mathrm{C}$. The PCR reaction was performed in duplo for each sample using nuclease-free water as the negative control.

\section{Enzyme-linked immunosorbent assay (ELISA)}

The protein expressions of HIF-1a, HIF-2a, Cygb, and PGC-1a were assayed by Mouse Elabscience ELISA Kit (China). The assays were performed according to the sandwich ELISA principle. The micro ELISA plate provided by the producer has been pre-coated with specific antibodies for each protein. A total of $100 \mu \mathrm{l}$ standard or sample was added to each pre-coated well and incubated for $90 \mathrm{~min}$ at $37^{\circ} \mathrm{C}$, followed by the addition of detection antibodies labeled with biotin for each protein. Another incubation was performed for 1 $h$ at $37^{\circ} \mathrm{C}$, followed by three washes with a washing buffer. The avidin-horseradish peroxidase conjugate was added to each well and incubated at $37^{\circ} \mathrm{C}$ for 30 min. After five serial washings with the same buffer, $90 \mu \mathrm{l}$ substrate solution was added to each well. After $15 \mathrm{~min}$ of incubation in the same condition, $50 \mu \mathrm{l}$ stop solution was added, and the color of the samples turned yellow. The ODs were measured spectrophotometrically at a wavelength of $450 \mathrm{~nm}$. The concentrations of proteins were calculated by extrapolation of the obtained OD from the standard curve.

\section{Statistical analysis}

Statistical analysis was performed using statistical package for the social science (SPSS) version 20.0 software. The distribution of all data 

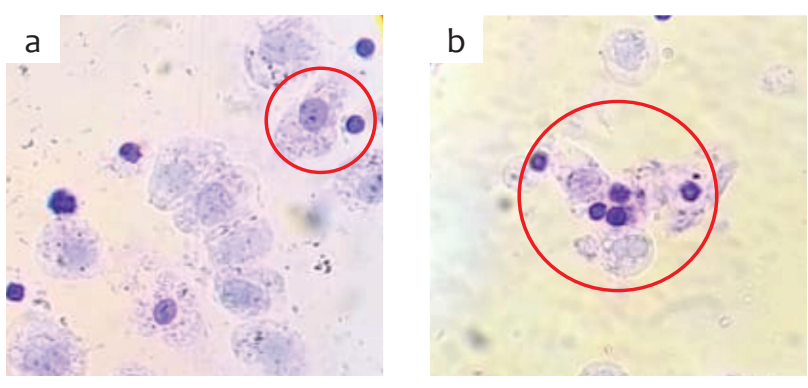

Figure 1. Phagocytosis by macrophages: (a) Macrophages began to engulf SRBC; (b) SRBC in the macrophages

was examined for homogeneity (Levine test) and normality (Shapiro-Wilk test), followed by analysis of variance and post-hoc test (parametric) or by Kruskal-Wallis and Mann-Whitney $U$ test (nonparametric).

\section{RESULTS}

\section{Phagocytosis experiment}

The phagocytosis experiment showed that the activated macrophages interacted with the SRBCs acting as antigen. LPS was administered in this experiment to increase macrophage activity. As shown in Figure 1a, after 15 min of incubation, the macrophage membrane began to bind to the SRBC membrane. Furthermore, after prolonged incubation, the SRBCs were compartmentalized in the macrophages (Figure 1b).

\section{mRNA expressions of HIF-1a, HIF-2a, and Cygb}

Figure 2 presents the HIF-1a mRNA expression. The HIF-1a mRNA expression slightly increased at 24

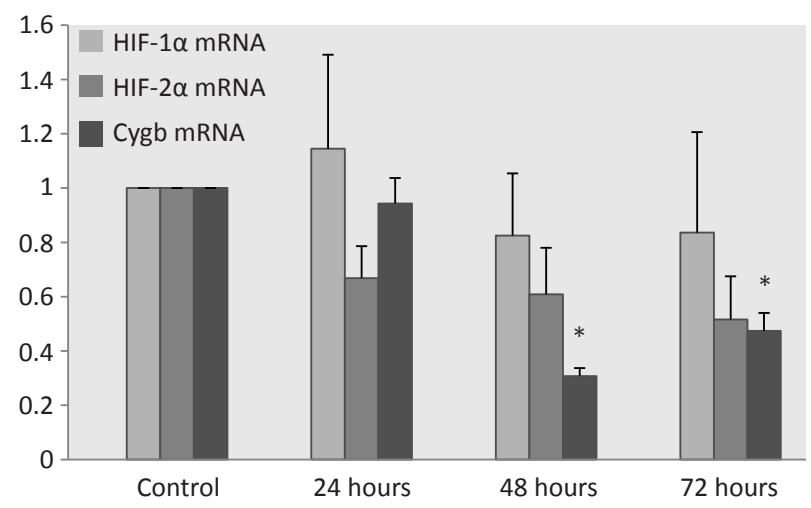

Figure 2. Relative expression of HIF-1a, HIF-2a, and Cygb mRNA in peritoneal macrophages of immunized BALB/c mice $*(p<0.005$ compared with the control group) hours after immunization and then decreased after 48 and 72 hours. No difference was observed between the immunization groups and the control group. Figure 2 also shows the HIF-2a mRNA expression. The HIF-2a mRNA expression decreased at 24,48 , and 72 hours after immunization compared with the control groups. No significant difference was noted between the immunization and control groups. Figure 2 displays the Cygb mRNA expression. The expression of Cygb mRNA decreased significantly at 48 and 72 hours after immunization compared with the control $(p=0.000)$.

\section{HIF-1a, HIF-2a, Cygb, and PGC-1a protein levels}

Figure 3 presents the HIF-1a protein expression. The figure shows that the protein levels were low at 24 hours after immunization but then increased after 48 and 72 hours $(p=0.000)$. Figure 3 shows the HIF- $2 a$ protein expression. The level of this protein was low at 24 hours but then increased after 48 and 72 hours $(p=0.035)$. Figure 3 also displays the Cygb protein expression. Compared with the control, the level of Cygb protein increased after 24, 48, and 72 hours, with the highest level observed at 72 hours after immunization $(p=0.010)$. Figure 3 also presents the $\mathrm{PGC}-1 a$ protein expression. The level of this protein was low at 24 hours but increased at 48 and 72 hours $(p=0.006)$.

\section{Superoxide anion concentration}

Figure 4 shows the OD results of WST reduction at $0,24,48$, and 72 hours after immunization. The OD value represents the concentration amount of the superoxide anion. The highest amount of superoxide



Figure 3. HIF-1a, HF-2a, Cygb, and PGC-1a protein in peritoneal macrophages of immunized BALB/c mice $*(p<0.005$ compared with the control group) 


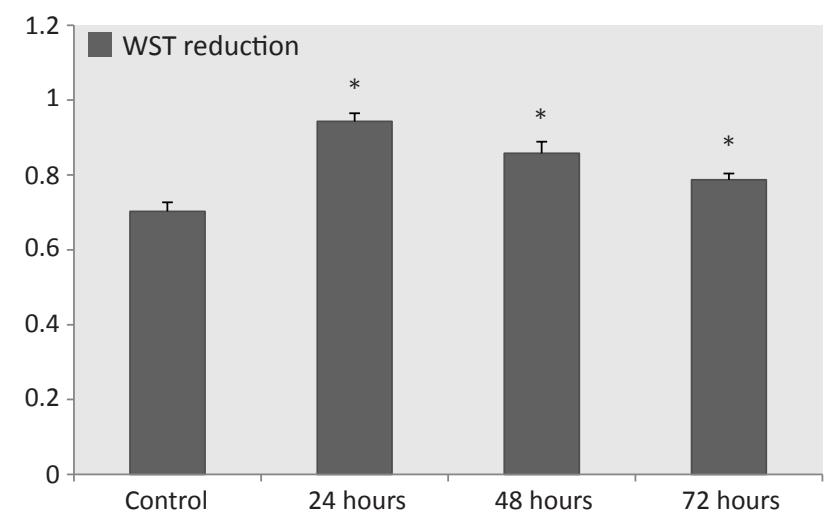

Figure 4. WST reduction by superoxide anions generated by the peritoneal macrophages of immunized BALB/c mice $*(p<0.005$ compared with the control group)

anion was observed at 24 hours after immunization. The level of superoxide anion decreased after 48 and 72 hours, but the level was still higher than that of the control group.

\section{DISCUSSION}

Active phagocytic cells exhibit a sharp increase in respiration, an event called the respiratory burst. ${ }^{14}$ Oxygen is needed to destroy the phagocytosed foreign bodies. As the oxygen supply is still the source, when a sudden increase in oxygen is needed, the phagocytic cells are in relative hypoxia. As indicated by Semenza et al,? the hypoxia state will be overcome at the gene level by the activity of a number of genes necessary to combat the situation. A transcription factor, i.e., HIF-1 was proven indispensable to regulating the genes. On the other hand, oxygen is converted into certain ROS, such as $\mathrm{O}_{2}, \mathrm{H}_{2} \mathrm{O}_{2}$, and $\mathrm{ClO}$. All these compounds pose danger but are needed to destroy the foreign bodies. Thus, the phagocytes themselves are threatened by their own ROS production. Therefore, to avoid failure, the phagocytes should undergo a process called oxygen burst.

The macrophages activated by SRBCs will engulf foreign bodies through oxidation and hydrolysis in phagosomes. In this study, the WST test provided high absorbance not only after 24 hours but also in the next 2 days. This condition indicates that the oxygen burst process was still in progress, finally triggering a hypoxic condition. Babior reported that peritoneal macrophages can produce superoxide anion 2 and 3 days after isolation. ${ }^{14}$ The oxygen burst usually occurred in the first 1 to 24 hours after. The positive WST reaction in this period indicated the formation of ROS in the form of superoxide anion. This radical is formed by the reduction of oxygen by nicotinamide adenine dinucleotide phosphate (NADPH), which is catalyzed by the membrane-bound enzyme NADPH oxidase. ${ }^{15}$

As a radical, superoxide anion shows no high reactivity and is immediately converted into hydrogen peroxide by superoxide dismutase, which is found in the cytoplasm..$^{16,17}$ The product, $\mathrm{H}_{2} \mathrm{O}_{2}$, is relatively non-hazardous except in the presence of a free iron $\left(\mathrm{Fe}^{2+} / \mathrm{Fe}^{3+}\right)$. The active macrophages and granulocytes contain another enzyme that reduces $\mathrm{H}_{2} \mathrm{O}_{2}$ with $\mathrm{Cl} / \mathrm{l}$. The enzyme, myeloperoxidase, produces a very potent oxidant, that is, a hypochlorite $(\mathrm{OCl})$ or hypoiodic $(\mathrm{Ol})$ molecule. In this manner, the macrophages can kill microorganisms. Therefore, in this study, oxygen demand was still high at 24 hours after immunization as macrophages require energy and antimicrobial substances to kill foreign bodies. However, the free radicals were generated as the increased oxygen demand can stimulate the expression of HIF-1a. ${ }^{18}$ This finding can explain the higher level of mRNA. However, in this period, the macrophages also actively synthesized and secreted several cytokine proteins.

All these processes need high energy, which can only be provided by increasing the energy metabolism. The high level of HIF-1a protein could also be caused by the inhibition of HIF-1a degradation. Normally, in the oxygen-sufficient conditions, HIF-1a is oxidized by prolyl hydroxylase domain (PHD), followed by degradation through the ubiquitin-protease system. However, in this case, although given the high level of oxygen, the macrophages remained in hypoxia conditions. This condition is a mechanism of adaptation to the oxygen burst process, which still occurred at 24 hours after immunization as evidenced by the WST test at 48 and 72 hours after immunization. However, the higher level of HIF-1a proteins not necessarily resulted from the presence of ROS or decreased hydroxylation. The ROS also led to PHD inactivation via the oxidation of ferrous iron in the catalytic domain, resulting in HIF- $a$ accumulation. ${ }^{19,20}$

In the more later period ( 48 and 72 hours), the levels of HIF-1a and HIF-2a were still high despite the low mRNA level of both proteins. This finding indicates that the hypoxic conditions still persisted owing to the still high levels of both HIFs. Both HIFs were not synthesized de novo, as indicated by the low level 
of correspondence mRNA. In this later period, the macrophages began to engage in adaptive immunity. At the same time, the antigen processed was presented at the surface of the cell membrane in the major histocompatibility complex II to the appropriate lymphocytes. The macrophages must synthesize several cytokine proteins in a high-energy-consumption process. When the macrophages are activated by foreign bodies or antigens, they secrete cytokines, such as tumor necrosis factor, interleukin (IL)-1, IL6, IL-8, and IL-12..$^{21,22}$ In another study, the secretion of cytokines involved mitochondrial activities. ${ }^{23}$ As is known, the high energy needed to be represented by ATP can only be fulfilled by aerobic metabolism, which needs oxygen and mitochondria as the site of the aerobic metabolism. This condition means that the macrophages require a type of molecule that can store and release oxygen. The need for such molecule is indicated by the presence of Cygb, which was detected in high levels during the observation period. This study has shown that Cygb is also under the control of HIF$1 a^{18}$

PGC-1a is reported as a marker of biogenesis of mitochondria. ${ }^{24}$ In our observation, the level of PGC1 was lower than that of the control at 24 hours and was much higher in the later period. We cannot explain why no biogenesis of mitochondria occurred in the 24 hours period despite the oxygen burst. However, the mechanism of high uptake of oxygen in the oxygen burst is totally independent of mitochondrial function. The uptake mechanism is facilitated by the membranebound NADPH oxidase. ${ }^{15}$ In the later period, the WST value was still high, and such finding was mainly caused by the active mitochondria. The WST positive reaction, which is based on the reduction, can be also caused by the active mitochondria, especially by specific intramitochondrial dehydrogenases..$^{25,26}$ The high level of PGC-1 itself also indicated the need for higher aerobic metabolism as the PGC-1a is a marker of the biogenesis or formation of new mitochondria. This study indicated that during an immune response, the macrophages underwent two types of stresses, i.e., hypoxia and oxidative stress. Both conditions need highly fine regulation and support from antioxidant and prooxidant substances. Such finding means that in any immune response, immunization, or infection, the subject needs appropriate nutrition support, which includes the antioxidant and prooxidant nutrition. This study is specific for peritoneal macrophages, that is, not all macrophages originated from all the tissues. Therefore, this study would provide better results if the macrophages were examined elsewhere, such as the lymphoid tissue. In addition, tissues that are always exposed to foreign objects, i.e., the lung tissues, also need to be examined, because they contain lung macrophages. Moreover, the lung tissues are very important as an entry point of tuberculosis and other airborne infectious diseases.

\section{Conclusions}

In general, this experiment suggests that the macrophages activated by SRBCs experience dual hypoxia and oxidative stress conditions to overcome the foreign bodies. The macrophages overcome these stress conditions by increasing their aerobic metabolism.

\section{Conflict of Interest}

PT. Ecosains Hayati provided PCR machines for gene expression analysis in this study. Sri Widia Jusman is one of the editorial board members but was not involved in the review or decision process of the article.

\section{Acknowledgment}

The author would like to acknowledge the head of DRPM UI who provided funds for this study.

\section{Funding Sources}

This study was funded by DRPM UI with research grant number: 1684/UN2.R12/HKP.05.00/2015.

\section{REFERENCES}

1. Wang C, Yu X, Cao Q, Wang Y, Zheng G, Tan TK, et al. Characterization of murine macrophages from bone marrow, spleen and peritoneum. BMC Immunol. 2013;14:6.

2. Weinberg JB. Mononuclear phagocytes. In (Lee GR, Foerster J, Luckens J, Paravekas F, Greer JP, Rodgers GM, eds). Wintroub's Clinical Haematology. Philadelphia: Lippincot Williams and Wilkins. 1998. p. 377-414.

3. Slauch JM. How does the oxidative burst of macrophages kill bacteria? still an open question. Mol Microbiol. 2011;80(3):580-3.

4. Iles KE, Forman HJ. Macrophage signaling and respiratory burst. Immunol Res. 2002;26(1-3):95-105.

5. de Duve C. The lysosome turns fifty. Nat Cell Biol. 2005;7(9):8479 .

6. Wang GL, Semenza GL. General involvement of hypoxiainducible factor 1 in transcriptional response to hypoxia. Proc Natl Acad Sci U S A. 1993;90(9):4304-8.

7. Semenza GL. Hypoxia-inducible factors in physiology and medicine. Cell. 2012;148(3):399-408.

8. Zhao C, Du W. Dynamic features of carboxy cytoglobin distal mutants investigated by molecular dynamics simulations. J Biol Inorg Chem. 2016;21(2):251-61.

9 Jusman SW, Halim A, Wanandi SI, Sadikin M. Expression of hypoxia-inducible factor-1a (HIF-1a) related to oxidative stress in liver or rats-induced by systemic chronic normobaric hypoxia. Acta Med Indones. 2010;42(1):17-23.

10. Liu H, Mao P, Wang J, Wang T, Xie CH. Allicin protects $\mathrm{PC} 12$ cells against 6-OHDA-induced oxidative stress and mitochondrial 
dysfunction via regulating mitochondrial dynamics. Cell Physiol Biochem. 2015;36(3):966-79.

11. Ray A, Dittel BN. Isolation of mouse peritoneal cavity cells. J Vis Exp. 2010;(35):1488.

12. Layoun A, Samba M, Santos M. Isolation of murine peritoneal macrophages to carry out gene expression analysis upon Tolllike receptors stimulation. J Vis Exp. 2015;(98):e52749.

13. Von V. Expressions-und funktionsanalysen von neuroglobin und cytoglobin. Dissertation. Mainz: Johanes GutenbergUniversitat; 2007.

14. Babior BM. The respiratory burst of phagocytes. J Clin Invest. 1984;73(5):599-601.

15. Cross AR, Segal AW. The NADPH oxidase of professional phagocytes-prototype of the NOX electron transport chain systems. Biochim Biophys Acta. 2004;1657(1):1-22.

16. Halliwell B, Gutteridge JM. Free radicals in biology and medicine. $3^{\text {rd }}$ Ed. London: Oxford University Press; 1999. In (Blanksby S, Ellison GB, Bierbaum Vm, Kato S, eds). Superoxide does react with peroxides: direct observation of the Haber-Weiss reaction in the gas phase. Angew Chem Int Ed Engl. 2007;46(26):494850.

17. McCord JM, Fridovich I. Superoxide dismutase. An enzymic function for erythrocuprein (hemocuprein). J Biol Chem. 1969;244(22):6049-55.

18. Jusman SW, Iswanti FC, Suyatna FD, Ferdinal F, Wanandi SI, Sadikin M. Cytoglobin expression in oxidative stressed liver during systemic chronic normobaric hypoxia and relation with
HIF-1a. Med J Indones. 2014;23(3):133-8.

19. Cash TP, Pan Y, Simon MC. Reactive oxygen species and cellular oxygen sensing. Free Radic Biol Med. 2007;43(9):1219-25.

20. Krock BL, Skuli N, Simon MC. Hypoxia-induced angiogenesis: good and evil. Genes Cancer. 2011;2(12):1117-33.

21. Aranggo Duque A, Descoteaux A. Macrophages cytokines: involvement in immunity and infectious diseases. Front Immunol. 2014;5:491.

22. Abbas AK, Lichtman AH, Pillai S. Cellular and molecular immunology. $6^{\text {th }}$ Edition. Philadelphia: Elsevier Publisher; 2007. p. 544

23. Yang D, Elner SG, Bian ZM, Till GO, Petty HR, Elner VM. Proinflammatory cytokines increase reactive oxygen species through mitochondria and NADPH oxidase in cultured RPE cells. Exp Eye Res. 2007;85(4):462-72.

24. D'Errico I, Salvatore L, Murzilli S, Lo Sasso G, Latorre D, Martelli N, et al. Peroxisome proliferator-activated receptor- $\gamma$ coactivator $1-\alpha(\mathrm{PGC}-1 \alpha)$ is a metabolic regulator of intestinal epithelial cell fate. Proc Natl Acad Sci U S A. 2011;108(16):66038.

25. Berridge MV, Herst PM, Tan AS. Tetrazolium dyes as tools in cell biology: new insights into their cellular reduction. Biotechnol Annu Rev. 2005;11:127-52.

26. Buzgariu W, Zarnescu O, Caloianu M, Coroiu V, Titescu GH, Stefanescu I. Ultrastructural and functional changes in mitochondria induced by heavy water in vitro. Rev Roum Biol. 2003;48(1-2);103-14. 